\title{
106 On the Fluorescence of Flour-spars Excited by Light of Different Wave-lengths.
}

By Hisamitu Nisı and Kyûitirô MrYamoto.

Kyushu Imperial University.

(Rec. July 10, 1928. Comm. by H. NAGAOKA, M.1.A., July 12, 1928.)

Fluor-spars may be conveniently classified into three groups, so far as we have examined the fluorescent spectra of fifteen crystals ${ }^{1}$ from different localities, - the first shows blue and red fluorescence, the second blue only, and the third no appreciable fluorescence. Here we deal with the first group only.

We chose a typical specimen (probably from Cumberland, England) from the group concerned, which is very clear and has purple color. It was polished in a cubic form and supported on a stand, just close to the slit of a (Hilger constant deviation) spectrograph, protected from stray light by suitable cover and screen. It was illuminated from the side by a large condensing quartz lens with the arc or spark between various metal electrodes.

The strong lines in the fluorescent spectra called forth by various exciting sources are given in the Table I, where the sensitive lines are emphasized, but the lines which are superposed on the blue fluorescence are indicated only for the case of $\mathrm{Ni}$-spark.

Table I.

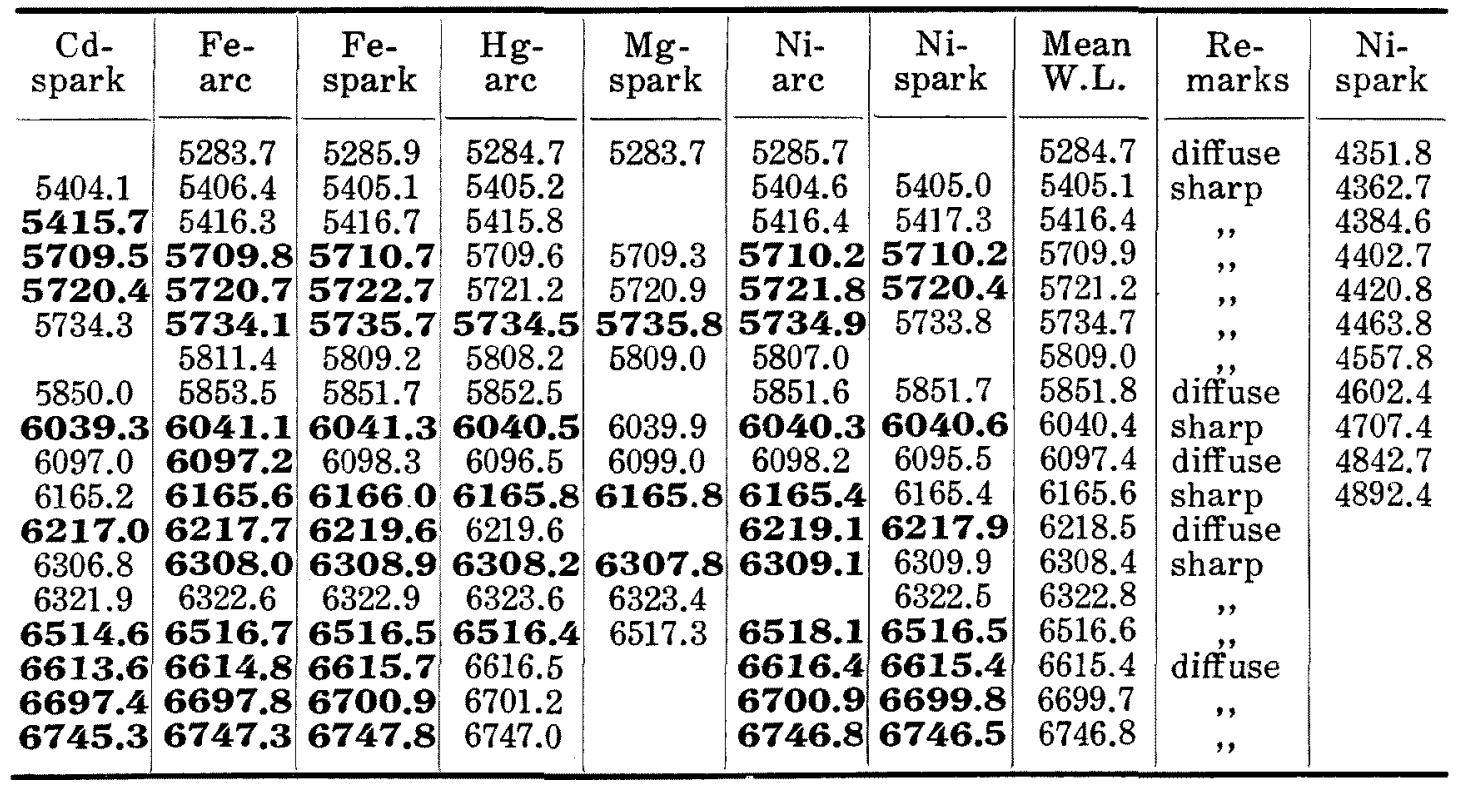

1) Some of them were obtained through the kindness of Prof. Kô, to whom our best thanks are due. 
Neon and nickel arc lines were used for comparison of wave-length. It should be noted the above results are not only characteristic of the specimen, but are confirmed in other seven specimens, although the fluorescent spectra vary with specimens more or less, probably owing to the difference of phosphors.

Generally speaking, the wave-lengths of definite fluorescent lines are constant within the experimental errors, whatever the exciting sources may be, and nearly constant for different specimens. This is the principal point, different from Morse's ${ }^{1)}$ earlier work. If the exciting source, such as $\mathrm{Ni}$ - or Fe-spark, contains many strong lines over the region from $3000 \mathrm{~A}$ to $2100 \mathrm{~A}$, nearly all the fluorescent lines are excited, although the intensity distribution varies from source to source; otherwise some of them disappear, as in the case of Mg-spark.

Fig. 1 and Fig. 2 are the spectra, excited by $\mathrm{Ni}$ - and Mg-sparks respectively.

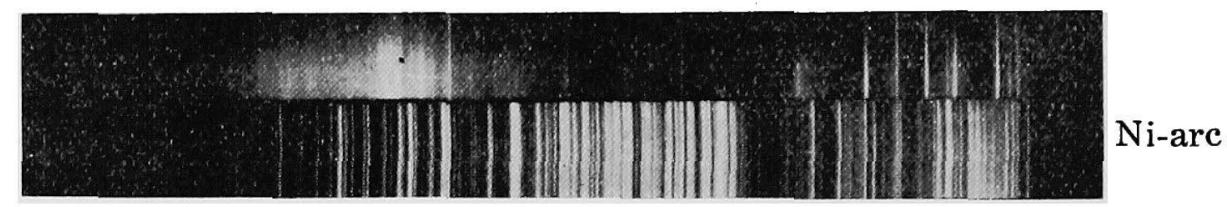

Fluorescent Spectrum of a Fluor-spar Excited by Ni-spark

Fig. 1.

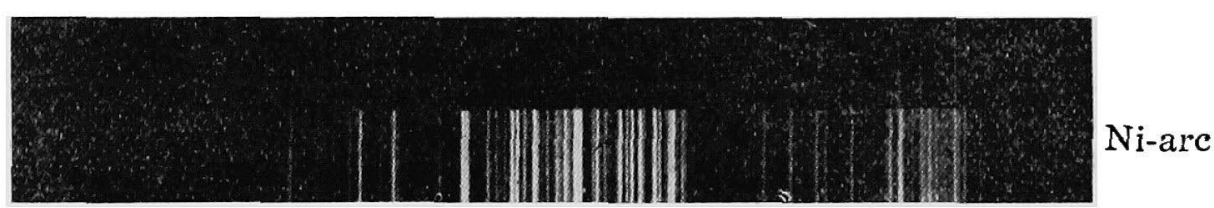

Fluorescent Spectrum of a Fluor-spar Excited by Mg-spark

Fig. 2.

As to the relation between the fluorescent spectra and the wavelengths of the exciting source may be mentioned:

Firstly, the blue fluorescence practically disappears, when Wratten aesculin filter or Wood's glass of about $2 \mathrm{~cm}$. is inserted between the specimen and the exciting source, but a thin Wood's glass has no appreciable effect upon it ; thus the principal wave-lengths able to excite the blue fluorescence is less than $3850 \AA$ and greater than $3000 \mathrm{~A}$. When excited by Mg-spark or light of wave-length with which the red fluorescence begins to appear, it becomes rather weak; but

1) Morse: Astrophys. Journ. 21 (1905), 83. 
with shorter wave-lengths (for ex. $2400 \AA$ ) its intensity again increases. For the latter case it should be taken into consideration that the lines and bands in the blue fluorescence have some influence upon the intensity, because they are so numerous and liable to overlap one another, if the slit is wide.

Secondly, to investigate the red fluorescence in detail, we took away the slits of collimator and telescope of a Hilger quartz monochromatic illuminator. A spark was put in place of the former and a slit of black paper was arranged for the latter from which the specimen was supported about $1 \mathrm{~cm}$. apart, in front of the colimator slit of a small spectrograph with direct vision prisms.

The exciting wave-lengths from $\mathrm{Fe}-, \mathrm{Ni}-$, and $\mathrm{Mg}$-spark were about $2300 \AA, 2500 \AA$, and $2800 \AA$, the allowance being $\pm 50, \pm 75$, and \pm 100 units respectively, measured by Beck ultra-violet spectroscope.

As the intensity of fluorescent light was very weak, it generally took more than 100 hours to take a photograph of fluorescent spectrum, although the slit was so wide that the closely situated lines were hardly resolved. Consequently it was difficult to distinguish two shorter ones of three lines in yellow from each other and we could not determine the wave-length so accurately; nevertheless the results are decidedly consistent and given in Table II.

Table II.

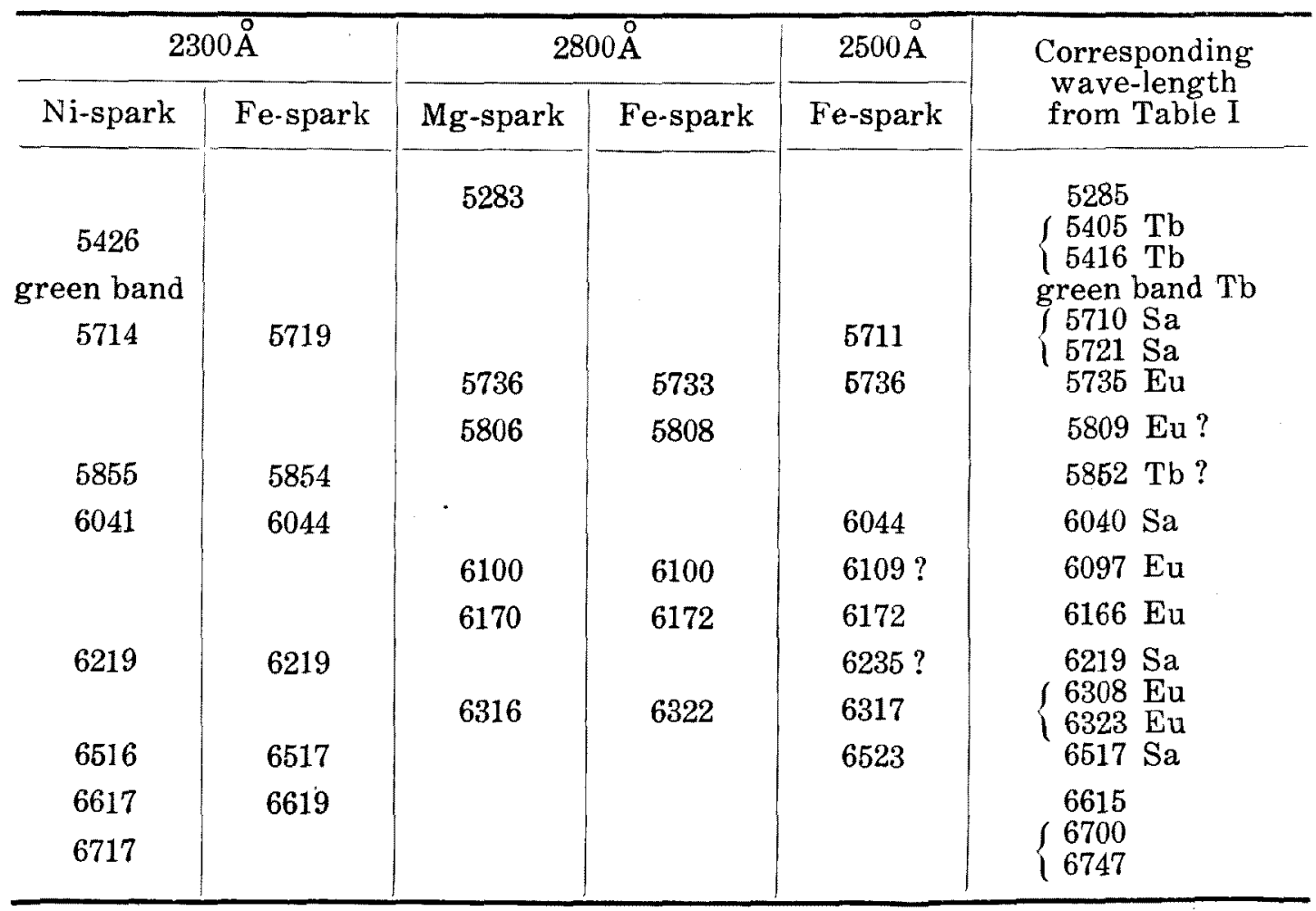


Fig. 3 and Fig. 4 are the reproduced photographs excited by $2300 \AA$ and $2800 \AA$ respectively.

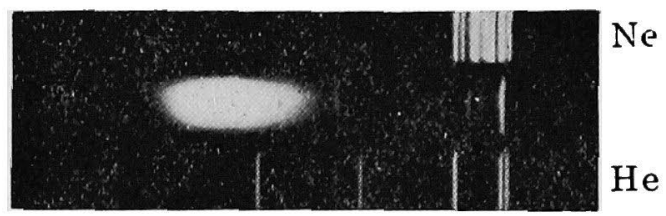

Fluorescent Spectrum of a Fluor-spar Excited by $2300 \AA$ Fig. 3.

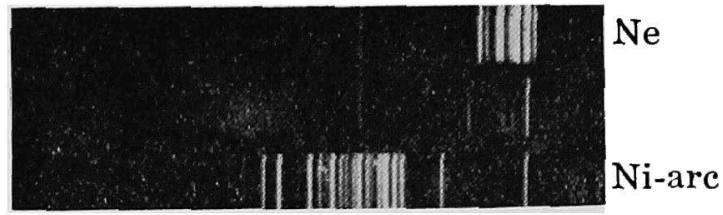

Fluorescent Spectrum of a Fluor-spar Excited by $2800 \AA$

Fig. 4.

The three lines strongly excited by Mg-spark appear feebly with Fe-arc, filtered by a thin Wood's glass. In other words, even the light of wave-length greater than $3000 \AA$ also takes part in exciting some lines in red fluorescence.

Thus we can divide the principal lines in the fluorescent spectrum into two main groups in which they are interlined against ordinary fluorescence. One group is excited by about $2300 \AA$ and consists of lines in red and yellow which are usally accompanied by the green fluorescence and lines in the blue fluorescence; the other by about $2800 \AA$. In this direction, the ultra-violet absorption of a fluor-spar has an intimate connection, but the specimen concerned does not show any distinct absorption bands in the region from $3000 \AA$ to $2100 \AA$ at ordinary temperatures. A definite fluorescent line seems to have its region of exciting wave-lengths, but not to be excited by a corresponding monochromatic light only. On the other hand, the lines which belong to each main group may form a series. As a matter of fact, if the wave-numbers of the lines which belong to $2800 \AA$-group are plotted as abscissae against successive integers, overlooking the difference in the properties of each line, the points seem to lie on a monotonic curve.

On comparing the results mentioned above with the well known works on the cathodo-luminescence of synthetic fluoride by Urbain ${ }^{1)}$ and others, we remark that most of the lines which belong to the $2300 \AA$-group are identified with those due to samarium phosphor and perhaps the lines belonging to the $2800 \AA$-group and those in green and blue fluorescence mostly originate from europium and terbium phosphors respectively. The difference in intensity and wave-length may be explained by the mutual influence of mixed phosphors. Thus the existence of rare earth phosphors is probable in the case of fluorescence.

1) Handbuch der Exp. Phys. Bd. XX. 1. Teil pp. 460-497. 